\title{
The Possible Influence of Microbiota on Food Compulsion
}

\author{
Rachel Arantes ${ }^{1}$, Emília Alves ${ }^{2}$, Manuel Bicho $^{3,4}$ and Ana Valente ${ }^{1,3 *}$ \\ ${ }^{1}$ Atlântica - Escola Universitária de Ciências Empresariais, Saúde, Tecnologias e Engenharia, Portugal \\ ${ }^{2}$ Escola de Ciências e Tecnologias da Saúde, Universidade Lusófona de Humanidades e Tecnologias, Portugal \\ ${ }^{3}$ Laboratório de Genética, Instituto de Saúde Ambiental, Faculdade de Medicina da Universidade de Lisboa, Portugal \\ ${ }^{4}$ Instituto de Investigação Científica Bento da Rocha Cabral, Portugal
}

\begin{abstract}
Introduction: The intestinal microbiota performs numerous functions in the body: appetite and satiety control, neurotransmitter production and other metabolites. The cepas that compose it are directly influenced by the diet. Certain microorganisms may act on dopamine neurocircuit towards a search for substances that promote the feeling of pleasure which leads to a compulsive addiction and impulsive behaviours as reward. There is plenty information about healthy foods and how to balance the diet to maintain health and longevity. However, it is still very difficult to change unhealthy eating patterns, and the patient is given full responsibility for those behaviours by being asked for "self-control" and "willpower". Is this the individual's own desire or is he stimulated by something else that he is not aware of?
\end{abstract}

Aim: Try to understand the relationship of the microbiota with its host, as well as the microorganisms present in the gut may induce the food preferences.

Methodology: For this review work, an extensive search was carried out using several online databases, namely SciELO, PubMed and Google Scholar. Research was also carried out on several websites of international and governmental organizations. Using the keywords "Obesity gut microbiota compulsive eating" produced 4,700 results between the years 2015 and 2019. In the present work, only articles and documents that could be consulted in their full content were used.

Conclusion: Alteration of the intestinal microbiota, mainly due to phylum imbalances, can lead to nutritional unbalance such as obesity, food dependence or compulsive eating. Although further studies are needed, to understand how the microbiota may play an important role in controlling eating disorders.

KEYWORDS: Obesity; Microbiota; Gut; Eating; Nutritional unbalance

Quick Response Code:

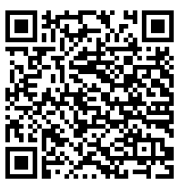

Address for correspondence: Ana Valente, Atlântica - Escola Universitária de Ciências Empresariais, Saúde, Tecnologias e Engenharia, Fábrica da Pólvora de Barcarena, Portugal

Received: June 02, 2020 Published: June 09, 2020

How to cite this article: Rachel A, Emília A, Manuel B, Ana Valente. The Possible Influence of Microbiota on Food Compulsion. 2020 - 2(3) OAJBS.ID.000179. DOI: 10.38125/OAJBS.000179 


\section{INTRODUCTION}

The growing pandemic of obesity has been the subject of studies worldwide. Data from the World Health Organization (WHO) indicate that approximately $60 \%$ of the population in developed countries is obese or overweight [1]. The relationship between obesity, cardiovascular diseases, diabetes, among others, is widely documented, which makes this condition one of the main public health problems today. In addition to physical comorbidities, obesity is also linked to psychological disorders such as low selfesteem, anxiety, depression and various eating disorders such as bulimia nervosa, night eating syndrome and Periodic Eating Compulsion Disorder (BED). Individuals with BED have greater difficulty in resisting foods rich in sugar and fat, as these offer a feeling of physical-emotional comfort [2]. While there is currently a lot of information about healthy foods and the best food choices to balance the diet and maintain health are widely publicized, breaking unhealthy eating patterns can be very challenging for this group of people. The patient is often given full responsibility for their eating behavior, encouraging them to have "self-control" and "willpower", which further weakens the individual's already fragile emotional state. However, it is worth asking whether this lack of self-control is an individual's difficulty or whether it is triggered by a stimulus that he himself ignores. The role of the intestinal microbiota in the host's eating behavior is an increasingly investigated topic, however the link between BED and the intestinal microbiota remains uncertain, and further studies are needed to help understand this topic [3]. The present work aims to contribute to understand the relationship between eating behavior and the individual's intestinal microbiota and how it may have an influence on the host's food preferences.

\section{MATERIALS AND METHODS}

For this review work, an extensive search was carried out using several online databases, namely SciELO, PubMed and Google Scholar. Research was also carried out on several websites of international and governmental organizations such as: American Society for Metabolic and Bariatric Surgery, Proceedings of the National Academy of Science of the United States of America, National Human Genome Research Institute, APC Microbiome Ireland SFI Research Center, World Health Organization and National Center of Biotechnology Information. To complement the information contained in this work, data obtained from international congresses in the field. When searching the online databases for the keyword "obesity", it produced more than 480 thousand results, when associating (obesity) with "intestinal microbiota" (gut microbiota) the result decreased to 35,200. Using the keywords, (Obesity gut microbiota compulsive eating) produced 4,700 results between the years 2015 and 2019. In order to understand the growing interest of the scientific community in this subject over the years, the last set of keywords was researched (Obesity gut microbiota compulsive eating), and time intervals in decades were used, beginning in the interval between the years 1960 to 1970, after 1970 to 1980 and so on. Generating the following numbers of articles: in the first decade 9 studies, in the second 58 studies, then 108 studies, then 454 studies, from 2000 to $2010,6,740$ studies and between 2010 and 2019 alone, 8,110 studies, more than all other decades combined. In the present work, only articles and documents that could be consulted in their full content were used. Totaling 50 scientific articles, 3 master's theses and 2 books.

\section{RESULTS AND DISCUSSION}

\section{Periodic Eating Compulsion Disorder- BED}

According to the fifth edition of the Diagnostic and Statistical Manual of Mental Disorders DSM-5 - American Psychiatric Association [4], binge eating is a disorder characterized by the ingestion of a large amount of food, in a short time, causing the only stop eating when feeling uncomfortably full. Feelings of guilt, shame and lack of control over the act of overeating are characteristic of BED. According to the DSM-5 pictures characterized by greed for specific foods, such as sweets and simple sugars, typical of depressive cases, are also common in individuals with BED. Patients with this type of disorder have episodes of binge eating, but do not use compensatory mechanisms to prevent weight gain, as is the case with individuals suffering from Bulimia Nervosa [5]. There are well-defined criteria for the diagnosis of BED. Binge eating episodes need to occur at least two days a week for the past six months, which are associated with uncontrolled food intake and that such behavior is not accompanied by compensatory behaviors to avoid weight gain [6]. It is worth noting that not all patients suffering from BED are overweight, but this is the most common disorder among obese people. Currently the great challenge among individuals with BED is to quantify what is an excessive consumption of food [7-9].

\section{Food Addiction}

Food addiction (AD), although not formally recognized by the DSM-5, is described in the scientific literature in pathologies such as eating disorders and obesity. The AD has a diagnostic tool, the Yale Food Addiction Scale - YFAS. This scale was developed in 2009 to identify those who are most likely to exhibit substance dependency markers such as consumption of foods high in fat, sugar or both. It is based on DSM-5 criteria for substance dependence, adapted for eating behavior. The diagnosis of food dependence is positive when it presents three or more symptoms, with clinically significant impairment or distress [10]. The concept of dependence on certain foods is still poorly understood by health professionals, because eating disorders are distinct from addictive behaviors and because the belief that "there are no bad foods, provided they are consumed in moderation", that makes it difficult to treat patients with BED. Some authors, such as Cotton et al. [11], concluded in studies with rats that, the intermittent and prolonged access to palatable foods makes the individual progressively avoid the less tasty diets and when the palatable foods become available again, they tend to overeat. He also observed anxiety behaviors during the period of abstinence from "addictive" foods. Pietiläinen et al. [12] said that the diet should not be restrictive, as it could perpetuate the disorderly eating cycle. For Manasse et al. [13], treatments for eating disorders fail because they do not address impulsivity as a central and determining factor in eating disorders. The increase in impulsivity and food-related compulsion plays a critical role in maintaining this disorder [14]. AD is described as an obesity phenotype that responds to reward [15]. However, it can exist without obesity and without BED [16]. About half of the patients with BED meet the AD criteria using the Yale Food Addiction Scale (YFAS), which suggests a deeper disturbance subtype than those with $\mathrm{BED}$ or $\mathrm{AD}$ alone [17]. AD can be antagonistic with weight loss efforts because it is a behavioral pathology, which interferes with the patient's tolerance and abstinence levels. Alterations in the dopamine circuit, observed in obese individuals, can make food less satisfying, and it is necessary to increase the amount and frequency of ingestion [18]. Highly processed foods have been more 
associated with food dependence [19]. Potenza [20], showed that the combination of refined white flour, sugar and fat, such as cookies and bread, is the most addictive combination and given the ease with which these products are acquired, desire and compulsion are important components to be considered in the context of eating disorders.

\section{Intestinal Microbiota}

The bacteria that make up the microbiota exceed 10 times the number of cells in the human body and 150 times the number of genes [21] and perform numerous functions in the body, such as: barrier against colonization by pathogenic microorganisms, protection and immune stimulation, anti-inflammatory properties, appetite and satiety control, production of vitamins and shortchain fatty acids, production of enzymes, among others [22]. The term "microbiota" refers to microorganisms such as bacteria, archaes, micro eukaryotes and viruses present in the human body, these function in commensal, symbiotic or pathological relationships with their host. The term "microbiome" refers to the set of genomes of these microorganisms [23]. Initially in the study of the microbiota, only culture methods were used, however, the feces as a sample, do not accurately represent the composition of the microbiota, which varies according to the anatomical portion of the intestine they colonize. In addition, due to the anaerobic nature of most gut bacteria, only 10 to $25 \%$ of these microorganisms were detected. Later, techniques based on RNA sequences, quantified the microbiome, but could not differentiate species. Currently, the combination of both techniques better reflects the diversity of bacteria belonging to the microbiota and allows a more detailed study. The method used to determine the microbiota must always be considered when planning studies and interpreting data [24].

\section{Composition of Healthy Human Microbiota}

Intestinal colonization, begins immediately after birth and its initial composition, will depend on the type of delivery, whether vaginal or cesarean and the feeding offered to the baby, whether breastfeeding or formula milk [25-26]. A healthy microbiota varies according to age, genetics, environment, diet and the structure of the intestinal wall [27]. The human intestinal microbiota has seven bacterial phyla: Firmicutes, Bacteroidetes, Actinobacteria, Proteobacteria, Fusobacteria, Verrucomicrobia and Cyanobacteria. Of these, the first three bacterial phyla are dominant.

\section{Alteration of the Microbiota by the Diet}

The intestinal microbiota is important for the energy balance [28]. Depending on the predominant macronutrient in the diet, specific species of bacteria are stimulated in the intestinal microbiota. Diets with a higher fat content are associated with an increase in bile-tolerant bacteria, such as Bacteroides, Alistipes and Bilophila. A high protein content will promote the development of butyrate-producing bacteria, specifically Clostridium cluster XVIa. In vegetarian and vegan diets, the intestinal microbiota is dominated by fermenting bacteria, such as Prevotella, Clostridium clostridioforme and Faecalibacterium prausnitzii [24]. The composition and function of the intestinal microbiota, in addition to varying according to the anatomical portion to which it is attached, is influenced by factors such as temperature, $\mathrm{pH}$, oxygen, water activity, salinity and light. Thus, throughout the gastrointestinal tract the distribution of the microbiota is not homogeneous, both in diversity and in quantity. The colon represents the most densely populated intestinal portion [29]. In Western food, the consumption of a large amount of foods rich in fat and simple carbohydrates (eg processed foods) and simultaneously low in fiber predominates, resulting in less diversity of the intestinal microbiota [30]. NonWestern diets, especially in rural areas, are characterized by a higher intake of dietary fiber and complex carbohydrates, which is reflected in the intestinal presence of a greater amount of polysaccharide fermenting bacteria, such as Prevotella, Succinivibrio and Treponema [21]. The microbiota regulates energy metabolism through the fermentation of undigested polysaccharides by the human body, resulting in short-chain fatty acids that represent about $10 \%$ of the daily energy consumed by humans [31]. These compounds are a source of energy for colonocytes and also serve as ligands for protein receptors, which can influence insulin sensitivity in adipocytes and peripheral organs, leading to less fat accumulation, improved intestinal motility, nutrient absorption and activation of the immune system [26,32].

\section{The Microbiota of Obese and Thin Individuals}

In obese people, the modification of the microbiota alters intestinal permeability (related to inflammatory processes), stimulates the absorption of monosaccharides and increases the intake of additional energy from the diet by increasing capillary density in the small intestine epithelium [33]. Excess calorie intake promotes the proliferation of bacteria from the phylum Firmicutes, favoring weight gain. On the other hand, other bacteria such as Bifidobacterium, Clostridium and Bacteroides have been identified as responsible for improving metabolism, the immune system, endocrine signaling and brain function [34]. One of the first studies on the subject carried out in animals, attempted to demonstrate the fundamental role of the intestinal microbiota in the host's energy metabolism, and it was found that rats with an absent microbiota (free of microorganisms) decreased considerably total body fat, despite the greater food intake, and returned to their normal state after colonization with a conventional intestinal microbiota [35]. In another study, colonization of mice without microbiota, with an obese human intestinal microbiota resulted in a substantial increase in total body fat compared to a lean intestinal microbiota [3]. However, although there is much evidence to indicate that the intestinal microbiota plays a key role in metabolic disorders, it has recently been shown that a high-fat diet is capable of generating obesity in rats, regardless of the intestinal microbial composition [36]. A recent meta-analysis demonstrated that the association between the intestinal microbial composition found in human feces and obesity is relatively weak [37]. In a study in obese humans, conducted by Duncan et al. [38]. concluded that the proportions of Bacteroidetes and Firmicutes have an important role in determining human obesity, however, it does not rule out the possibility that more detailed analyzes reveal changes in species composition between obese and thin individuals. To investigate the relationship between microbiota and body fat in humans, Ley et al. [39] studied 12 obese individuals randomly divided into two groups. One group received a low-calorie diet with fat restriction and the other a low-calorie diet with restriction of carbohydrates. The composition of the microbiota was monitored for one year. Despite the interpersonal differences, Bacteroidetes and Firmicutes dominated the microbiota $(92.6 \%)$. Before the diet, obese individuals had fewer Bacteroidetes and more Firmicutes compared to lean controls. Over time, the relative abundance of Bacteroidetes increased and the abundance of Firmicutes decreased, regardless of the type of diet. In this change determined by the diet, the occurrence or extinction of certain bacterial species did not occur, 
and the diversity remained constant over time. It was found that the increased amount of Bacteroidetes was related to the percentage of body weight loss and not to the type of macronutrient restricted in the diet. The intestine of the obese due to unknown factors seems to favor the Firmicutes [39]. The differences found between the conclusions of Duncan et al. [38] and Ley et al. [39], may be justified by the methodology used: the type of diet, the group of volunteers, the methods of preparing the samples and their storage or, still, the detection methods used. The intestinal flora of both humans and animals can be modified through the diet, and the intake of excess calories promotes the proliferation of bacteria from the phylum Firmicutes, which allow the extraction of nutrients with greater efficiency. Oral transplantation of Firmicutes in axenic rats (without microorganisms) causes obesity in these animals. The manipulation of both diet and intestinal flora may represent a new strategy for the treatment of obesity and its complications [40].

\section{Possible Mechanisms of Influence of the Microbiota on the Human Organism}

A good example of how a microorganism can manipulate its host and modify its behavior to an unnatural point, was the study by Vyas et al. [41]. In this study, rats were inoculated with toxoplasma gondi, which causes toxoplasmosis. The cat being the permanent host of this microorganism, once in the intestine of the studied rats, they modify their natural behavior, of aversion and escape in the presence of cat odor, to a behavior opposite to their nature, becoming attracted or with less fear of cats [41]. Some microbes can produce neurotransmitters [42]. Noraepinephrine can be produced by Escherichia, Bacillus and Saccharomyces, while serotonin can be produced by Streptococcus, Escherichia, Enterococcus and dopamine by Bacillus and Serratia. Other studies have shown that Bifidus Infantis increases plasma tryptophan concentrations and modulates 5-hydroxy-tryptamine (5-HT), a precursor to serotonin. Lactobacillus rhamnosus, on the other hand, alter the gammaaminobutyric acid receptor. Bravo et al. [43] found that there is less cortisol secretion in rats subjected to stress, supplemented with L. rhamnosus compared to a control group. These microorganisms have been identified as "psychobiotics because when ingested in adequate quantities, they produce positive psychiatric effects in several psychopathologies [44,45], state that dopamine is predominant in the brain, and is responsible for numerous functions such as locomotor activity and motor control, cognition, emotions, positive reinforcement, food intake and some endocrine functions. The dysfunction of the dopamine receptor leads to the search for substances that promote a sensation of pleasure, such as drugs, alcohol or food, generating addictive, compulsive and impulsive behaviors by valuing the reward. However, it is not clear whether the behavior is a cause or a consequence of the decreased response in the reward system [19]. There are many aspects to be unveiled regarding the action of microorganisms in the human organism. Their performance can be through their own constituents, such as DNA, peptidoglycans, lipopolysaccharides and flagellin, or through their metabolites. Some of these microbial metabolites have been attributed neuroactive properties, mainly to butyric, propionic and acetic acid. Several neurotransmitters are produced by commensal species, namely, serotonin, gammaaminobutyric acid, catecholamines, acetylcholine and histamine [46]. Possibly, the microbiota and its metabolites modulate brain behaviors and circuits that respond to stress, emotional behavior, food consumption, pain modulation and brain biochemistry [47]. Dinan et al. [48], describe the communication between the intestine and the brain by different routes [49]: through nerve communications of the microbiota with the vagus nerve, hypothalamic-pituitary-adrenal axis, which responds to stress, tryptophan metabolism, essential amino acid and precursor of the neurotransmitter serotonin, production of short chair fatty acids. Although the relationship between the intestinal microbiota and mental disorders is complex, it is possible to improve depression and anxiety through the modulation of the microbiota [50].

\section{Manipulation of the Microbiota as Control of Anxiety and Compulsive Eating}

Individual variation in dopamine neurocircuits can trigger opportunistic nutrition and consequent obesity. Dopamine plays a central role in associations of food reward, action and outcome, and routine or automated behaviors. Therefore, if obesity is related to habitual excesses, an active response with dopamine signaling is expected [51]. Another mechanism of dopamine that may be altered in obesity status is the hypofunction of the reward. In this condition, obese individuals eat too much to compensate for the attenuated reward signal [18]. It is therefore expected that the intestinal microbiota has an interest, from an evolutionary point of view, in modifying the host's eating behavior. Different bacteria have different nutritional needs, for example, Prevotella thrives on carbohydrates and Bacteroides seem to prefer animal proteins and fats. Alcock et al. [52] proposes the hypothesis that the population variety of the dominant microbiota is that it modulates the food intake in the host, imposing a certain selective pressure, leading to a positive feedback cycle, which possibly directs to specific food preferences and or patterns, in order to maintain a certain group of bacteria. Although studies in humans are not yet conclusive, data in animals point to some hypotheses. First, the impact of intestinal bacteria on hormonal production or activity that regulates appetite must be taken into account. Enteroendocrine cells, when activated by binding to bacterial metabolites, modify the secretion of hormones that regulate satiety and hunger [53]. Another relevant mechanism is the production of peptides that are analogous to hormones that regulate mammalian appetite. These peptides can mimic the hormone or trigger an autoimmune response that interferes with appetite regulation, causing the host to produce antibodies to fight microbial peptides, but because they are analogous, it ends up neutralizing the host's own hormonal effect. This mechanism is particularly important in the pathogenesis and evolution of eating disorders. Bacteria that produce neurotransmitters and neuromodulators [22], act directly on afferent axons or interact with intestinal epithelial cells and, therefore, with the enteric nervous system to modify neural signaling to the central nervous system [54]. Food restriction in patients with eating disorders alters the availability of energetic substrate (type, quantity and duration) for intestinal microbes and leads to different microbial profiles. Limited food choice is a direct selective pressure, as different microbes have their preferred substrates, for example, Roseburia and Bacteroides are sensitive to dietary carbohydrates and proteins, respectively, and the proportion of Bacteroides depends on the type of dietary fiber reaches the colon $[21,55,56]$. There seems to be some evidence that the microbiota may play an important role in the establishment, evolution and treatment of eating disorders, and it is now possible to use the beneficial properties of these microorganisms in a complementary way [31]. Several data ensure psychological benefits of manipulating the intestinal microbiota through probiotics $[44,57,58]$, namely in the reduction of anxiety and depression symptoms, showing to be a good adjunct in treatments for eating disorders [47,59]. Although the role of intestinal microbes in the host's eating behavior is 
being studied at an accelerated rate, the link between compulsive addiction and impulsive behaviors and the intestinal microbiota remains uncertain and further studies are needed [3].

\section{CONCLUSION}

The alteration of the intestinal microbiota or dysbiosis, determined by imbalances between phyla, can lead to disease (eg obesity), food addiction or BED. The delicate symbiosis between microorganisms and host, can generate mutual benefits as well as competition and manipulation. Microbes can also compete with each other. More diverse microorganism populations compete for more resources than less diverse bacterial populations Variations in dopamine neurocircuits can play an important role in the development of obesity, by potentiating opportunistic food intake in an obesogenic environment, through already known mechanisms of action of the intestinal microbiota in the context of metabolic diseases and changes in the host-microbe dynamics present eating disorders. It is important to assess the nutritional modulation of the patient's eating habits in order to restore the balance of the intestinal microbiota and the consequent impact on the modification of eating behaviours. Further studies are needed to better understand how the intestinal microbiota can affect the human host's health and its specific role in the appearance and development of eating disorders.

\section{REFERENCES}

1. (2016) World Health Organization. Global Health Observatory.

2. Stunkard A, Berkowitz R, Wadden T (1996) Binge eating disorder and night - eating syndrome. Int J Obes 20:1-6.

3. Wouw M, Schellekens H, Dinan T (2017) Microbiota-gut-brain axis: Modulator of host metabolism and appetite J Nutr 147(5): 727-745.

4. American Psychiatric Association (2014) Manual diagnostico e estatístico de transtornos mentais DSM-5 (5o ed.). Associação Brasileira de Psiquiatria, Artmed, Canada.

5. Appolinário J, Claudino A (2000) Transtornos alimentares. Braz J Psychiatry 22(Supl II): 28-31.

6. Cortez C, Araújo E, Ribeiro M (2011) Transtorno de compulsão alimentar periódico e obesidade. Rev Assoc Med Bras 40: 1.

7. Moreira R, Batista A (2007) Insatisfação com a imagem corporal em mulheres obesas: a importância do transtorno da compulsão alimentar periódica. Rev Psiquiatr Rio Gd Sul 29: 131-132.

8. Hudson JI, Hiripi E, Pope HG, (2007) The prevalence and correlates of eating disorders in the national comorbidity survey replication. Biol Psychiatry 61(3): 348-358.

9. Kessler RC, Berglund PA, Chiu WT (2013) The prevalence and correlates of binge eating disorder in the World Health Organization world mental health surveys. Biol Psychiatry 73(9): 904-914.

10. Pursey K, Stanwell P, Gearhardt A (2014) The prevalence of food addiction as assessed by the Yale Food Addiction Scale: a systematic review. Nutrients 6(10): 4552-4590.

11. Cotton P, Sabino V, Roberto M (2009) CRF system recruitment mediates dark side of compulsive eating. PNAS 106(47): 20016-20020.

12. Pietiläinen K, Saarni S, Kaprio J (2012) Does dieting make you fat? A twin study. Int J Obes 36: 456-464.

13. Manasse S, Espel H, Schumacher L (2016) Does impulsivity predict outcome in treatment for binge eating disorder? A multimodal investigation. Appetite 105: 172-179.

14. Schulte EM, Grilo CM, Gearhardt AN (2016) Shared and unique mechanisms underlying binge eating disorder and addictive disorders. Clin Psychol Rev 44: 125-139.
15. Davis C (2013) Compulsive overeating as an addictive behaviour: overlap between food addiction and binge eating disorder. Curr Obes Rep 2(2): 171-178.

16. Eichen D, Lent M, Goldbacher E (2013) Exploration of "food addiction" in overweight and obese treatment-seeking adults. Appetite 67: 22-24.

17. Gearhardt A, White M, Masheb R (2012) An examination of the food addiction construct in obese patients with binge eating disorder. Int J Eat Disord 45(5): 657-663.

18. Guo J, Simmons W, Herscovitch P (2014) Striatal dopamine D2-like receptor correlation patterns with human obesity and opportunistic eating behaviour. Mol Psychiatry 19: 1078-1084.

19. Schulte C, Molz S, Appelbaum S (2015) miRNA-197 and miRNA-223 predict cardiovascular death in a cohort of patients with symptomatic coronary artery disease. PloS one 10(12): e0145930.

20. Potenza M, Grilo C (2014) How relevant is food craving to obesity and its treatment? Front Psychiatry 5(164): 1-5.

21. Maukonen J, Saarela M (2015) Human gut microbiota: Does diet matter? P Nutr Soc 74(1): 23-36.

22. Nicholson JK, Holmes E, Kinross J, Burcelin R, Gibson G, et al. (2012) Host-gut microbiota metabolic interactions. Science 336(6086): 1262 1267.

23. Hollister E, Gao C, Versalovic J (2014) Compositional and functional features of the gastrointestinal microbiome and their effects on human health. Gastroenterology 146(6): 1449-1458.

24. Alou M, Lagier J, Raoult D (2016) Diet influence on the gut microbiota and dysbiosis related to nutritional disorders. Hum Microbiome J 1: 3-11.

25. Palmer C, Bik E, DiGiulio D (2017) Development of the human infant intestinal microbiota. PLoS Biol 5(7): 77.

26. Boulangé C, Neves A, Chilloux J (2016) Impact of the gut microbiota on inflammation, obesity and metabolic disease. Genome Med 8(1): 42.

27. Derrien M, Vileg H (2015) Fate, activity and impact of ingested bacteria within the human gut microbiota. Trends Microbiol 23: 6 .

28. Graf D, Di Cagno R, Fak F (2015) Contribution of diet to the composition of the human gut microbiota. Microb Ecol Health Dis 26: 26164.

29. WGO (2010) Probióticos e prebióticos - Directrizes Mundiais da Organização Mundial de gastroenterologia. World Gastroenterology.

30. Pistelli G, Costa C (2010) Bactérias intestinais e obesidade. Rev Saude Pes 3(1): 115-119.

31. Currò D, Ianiro G, Pecere S, Bibbò S (2017) Probiotics, fibre and herbal medicinal products for functional and inflammatory bowel disorders. Brit J Pharmacol 174(11): 1426-1449.

32. Wolf K, Lorenz R (2012) Gut microbiota and obesity. Curr Obes Rep 1(1): 1-8.

33. Lau E, Carvalho D, Pina-Vaz C (2015) Beyond gut microbiota: understanding obesity and type 2 diabetes. Hormones 14: 358-369.

34. Penteado J, Salgado R, Barlem E (2017) A eficácia do tratamento da obesidade através do transplante da microbiota fecal de indivíduos magros. Vittalle J Health Sci 29(1):46-53.

35. Bäckhed F, Ding H, Wang $\mathrm{T}$ (2004) The gut microbiota as an environmental factor that regulates fat storage. Proc Natl Acad Sci 101(44): 15718-15723

36. Rabot S, Membrez M, Blancher F (2016) High fat diet drives obesity regardless the composition of gut microbiota in mice. Sci Rep 6: 32484.

37. Sze MA, Schloss PD (2016) Looking for a signal in the noise: Revisiting obesity and the microbiome. mBio 7: 4 .

38. Duncan S, Lobley G, Holtrop G (2008) Human colonic microbiota associated with diet, obesity and weight loss. Int J Obes 32: 1720-1724.

39. Ley RE, Bäckhed F, Turnbaugh P (2005) Obesity alters gut microbial ecology. Proc Natl Acad Sci 102(31): 11070-11075. 
40. Yazigi A, Gaborit B, Nogueira JP (2008) Rôle de la flore intestinale dans l'insulinorésistance et l'obésitéRole of intestinal flora in insulin resistance and obesity. Presse Med 37(10): 14271430.

41. Vyas A, Seon-Kyeong K, Giacomini N (2007) Behavioral changes induced by toxoplasma infection of rodents are highly specific to aversion of cat odors. Proc Natl Acad Sci 107: 1731-1735.

42. Lyte M (2011) Probiotics function mechanistically as delivery vehicles for neuroactive compounds: Microbial endocrinology in the design and use of probiotics. Bioessays 3(8): 574-581

43. Bravo JA, Forsythe P, Chew MV (2011) Ingestion of Lactobacillus strain regulates emotional behavior and central GABA receptor expression in a mouse via the vagus nerve. Proc Natl Acad Sci 108(38): 16050-16055.

44. Sarkar A, Lehto S, Timothy S (2016) Psychobiotics and the manipulation of bacteria-gut-brain signals. Trends Neurosci 39(11): 763-781.

45. Sousa A, Fortunato J, Pinto J (2002) Receptors da dopamina e esquizofrenia. Rev Port Psicosso 4: 2.

46. Silvestre C (2015) 0 diálogo entre o cérebro e o intestino qual o papel dos probióticos? (Mestrado Integrado em Medicina). Faculdade de Medicina da Universidade de Lisboa.

47. Mayer E, Tillisch K, Gupita A (2015) Gut/brain axis and the microbiota. J Clin Invest 125(3): 926-938.

48. Dinan T, Stilling R, Stanton C (2015) Collective unconscious: how gut microbes shape human behavior. J Psychiatr Res 63: 1-9.

49. Landeiro J (2016) Impacto da microbiota intestinal na saúde mental. (Mestrado Integrado em Ciências Farmacêuticas). Instituto Superior de Ciências da Saúde Egas Moniz.
50. Liu X, Cao S, Zhang X (2015) Modulation of gut microbiota-brain axis by probiotics, prebiotics, and diet. J Agric Food Chem 63(36): 7885-7895.

51. Ribeiro G, Santos O (2013) Recompensa alimentar: mecanismos envolvidos e implicações para a obesidade. Rev Port Endocrinol Diabetes Metab 8(2): 82-88.

52. Alcock J, Maley C, Aktipis A (2014) Is eating behavior manipulated by the gastrointestinal microbiota? Evolutionary pressures and potential mechanisms. Bio Essays 36(10): 940-949.

53. Raybould H (2010) Gut chemosensing: interactions between gut endocrine cells and visceral afferents. Auton. Neurosci-Basic 153(12): 41-46.

54. Lam Y, Maguire S, Palacios T (2017) Are the gut bacteria telling us to eat or not to eat? Reviewing the role of gut microbiota in the etiology, disease progression and treatment of eating disorders. Nutrients 9: 602 .

55. Chung WS, Walker AW, Louis P (2016) Modulation of the human gut microbiota by dietary fibres occurs at the species level. BMC Biol 14: 3 .

56. Dominianni C, Sinha R, Goedert JJ (2015) Sex, body mass index, and dietary fibre intake influence the human gut microbiome. PloS one 10(4): e0124599.

57. Saulnier DM, Ringel Y, Heyman MB (2013) The intestinal microbiome, probiotics and prebiotics in neurogastroenterology. Gut Microbes 4(1): 17-27.

58. Ringel Y, Quigley EMM, Lin, HC (2012) Using probiotics in gastrointestinal disorders. Am J Gastroenterol Suppl 1: 34-40.

59. Huang R, Wang K, Hu J (2016) Effect of Probiotics on Depression: A Systematic Review and Meta-Analysis of Randomized Controlled Trials. Nutrients 8(8): 483 